\title{
Relationship Between Power Loss and Network Topology in Power Systems
}

\author{
Javad Lavaei and Steven H. Low
}

\begin{abstract}
This paper is concerned with studying how the minimum power loss in a power system is related to its network topology. The existing algorithms in the literature all exploit nonlinear, heuristic, or local search algorithms to find the minimum power loss, which make them blind to the network topology. Given certain constraints on power level, bus voltages, etc., a linear-matrix-inequality (LMI) optimization problem is derived, which provides a lower bound on the minimum active loss in the network. The proposed LMI problem has the property that its objective function depends on the loads and its matrix inequality constraint is related to the topology of the power system. This property makes it possible to address many important power problems, such as the optimal network reconfiguration and the optimal placement/sizing of distributed generation units in power systems. Moreover, a condition is provided under which the solution of the given LMI problem is guaranteed to be exactly equal to the minimum power loss. As justified mathematically and verified on IEEE test systems, this condition is expected to hold widely in practice, implying that a practical power loss minimization problem is likely to be solvable using a convex algorithm.
\end{abstract}

\section{INTRODUCTION}

The optimal power flow (OPF) problem deals with finding an optimal operating point of a power system that satisfies certain constraints on power and voltage parameters and, in addition, minimizes an appropriate cost function, such as generation cost or transmission loss [1]. The OPF problem has been extensively studied for several decades and numerous gradient and nonlinear algorithms have been proposed to find a near-optimal solution of this highly nonconvex problem [2], [3], [4], [5]. Since the power losses occurring in the transmission lines of a low-voltage distribution network are significant in comparison to the total amount of the power to be delivered, the power loss minimization problem, as a particular type of the OPF problem, has attracted much attention in the literature since 1962.

Power loss is minimized in a distribution network by means of various techniques, some of which are: (i) exploitation of capacitor banks, (ii) network reconfiguration and (iii) installation of distributed generation units. Method (i) relies on the fact that capacitor banks compensate for the reactive powers at the load buses, which could accordingly reduce the active power loss. The paper [6] employs a genetic algorithm to find the optimal places of the capacitors. In contrast, Method (ii) only modifies the configuration of the radial distribution network to diminish the power loss. Finding an optimal configuration leads to an OPF problem

Javad Lavaei is with the Department of Control and Dynamical Systems, California Institute of Technology (email: lavaei@cds.caltech.edu).

Steven H. Low is with Computer Science and Electrical Engineering Departments, California Institute of Technology (email: slow@caltech.edu). mixed with a graph combinatorial problem, which turns out to be extremely hard to solve. The papers [7], [8], [9] propose heuristic, meta-heuristic and fuzzy algorithms to find an optimal configuration. Motivated by the availability of renewable resources such as hydro, wind, solar, biomass and ocean energy, distributed generation (DG) units, e.g. microturbine, fuel cell, mini-hydro, battery storage, are becoming an important part of power systems. Method (iii) minimizes the power loss by integrating DGs in a distribution network. The problem of finding the optimal locations (placement) and values (sizing) of the DGs have been recently studied in many papers [10], [11], [12], [13].

To address the loss minimization problem using each of Methods (i), (ii) or (iii), it is crucial to understand the relationship between the power loss and the network topology. To this end, the present paper proposes a linearmatrix-inequality (LMI) optimization problem (a special type of convex optimization) that yields a nonnegative lower bound on the power loss [14]. This LMI problem has the remarkable property that its feasibility region only depends on the network topology, and the load profiles (as well as voltage magnitudes) just appear in the objective function. This decomposition property of the proposed LMI problem opens up the possibility of designing convex, numerically efficient algorithms to achieve an optimal placement of capacitor backs, optimal network reconfiguration or even optimal placement/sizing of DGs. Interestingly, the LMI problem given here attains the exact value of the loss (as opposed to a lower bound on it) for all IEEE test systems with 14, 30, 57, 118 and 300 buses. We connect this observation to the recent results on rank minimization and justify it rigorously [16], [17]. In fact, we show that a power system has certain physical properties, which can make a general OPF problem efficiently solvable.

The paper is organized as follows. The problem is formulated in Section II. The results are developed for a radial network in Sections III and IV, and then extended to a general network in Section V. Simulation results are provided in Section VI. Finally, some concluding remarks are drawn in Section VII.

Notations: We introduce the following notations:

- i : The imaginary unit.

- $\mathbf{R}$ : The set of real numbers.

- $\operatorname{Re}\{\cdot\}$ and $\operatorname{Im}\{\cdot\}:$ The operators returning the real and imaginary parts of a complex matrix.

- * : The conjugate transpose operator.

- $T$ : The transpose operator.

- $\succeq$ : The matrix inequality sign in the positive semidef- 
inite sense [14].

- $\|\cdot\|_{*}$ : The nuclear-norm operator (taking the sum of the singular values of a matrix).

\section{PROBlem Formulation}

Consider a power system with $m$ load buses and $n-m$ generator buses. With no loss of generality, assume that the power network consists of only transmission lines (and no transformers), where

- The resistance of every transmission line is strictly positive.

- The power network is a strongly connected graph.

Figure 1 illustrates a transmission system with three generator buses and three load buses, in which

- Each generator is represented by a circle.

- Two types of loads are connected to each of buses 1, 2 and 3: (i) constant-impedance loads shown by shunt admittances (rectangles), (ii) constant-power loads shown by triangles.

For every $k \in\{1,2, \ldots, n\}$, let $y_{k}$ denote the admittance of the constant-impedance load connected to bus $k$ and $V_{k}$ represent the complex voltage of this bus. Each transmission line in the network can be described by its equivalent $\Pi$ model. To be more precise, a transmission line connecting arbitrary buses $i, j \in\{1,2, \ldots, n\}$ (shown in Figure 2(a)) can be replaced by its $\Pi$ model given in Figure 2(b), where $z_{i j}$ and $y_{i j}$ denote the series impedance and shunt admittance, respectively. Define the admittance matrix $Y$ of the network as an $n \times n$ complex-valued matrix whose $(i, j)$ entry, $i, j \in$ $\{1,2, \ldots, n\}$, is given as:

$(i, j)$ entry of $Y=\left\{\begin{array}{cl}-\frac{1}{z_{i j}} & \text { if } i \neq j \\ y_{i}+\sum_{j \in \mathcal{N}(i)}\left(\frac{y_{i j}}{2}+\frac{1}{z_{i j}}\right) & \text { if } i=j\end{array}\right.$

where $\mathcal{N}(i)$ is the set of those buses that are connected to bus $i$. Note that the matrix $Y$ captures the topology of the power network and plays the role of the Laplacian of the weighted graph associated with the network. Stack the voltages $V_{1}, V_{2}, \ldots, V_{n}$ in a column vector, denoted by $V$. Define the current vector $I$ as $Y V$ and denote its $i^{\text {th }}$ element with $I_{i}$ for every $i \in\{1,2, \ldots, n\}$. It is easy to show that the complex power injected at a generator bus $j \in\{m+1, \ldots, n\}$ by its generator is equal to $V_{j} I_{j}^{*}$ and that the complex power consumed at a load bus $k \in\{1,2, \ldots, m\}$ by its constantpower load is equal to $-V_{k} I_{k}^{*}$.

Given some desired constant-power loads at the load buses, there may exist several ways to provision the generators' powers to supply such loads. Hence, it is important to find a set of generators' powers which not only minimizes the active/reactive power loss in the network, but also meets other practical constraints on voltages, powers, etc. This aim has been long studied in the context of optimal power flow problem, which is notorious for its high non-convexity. Note that active and reactive power losses occur in a power system due to the fact that transmission lines in the network have resistive, inductive and capacitive components. The objective of this paper is to study how the power loss is related

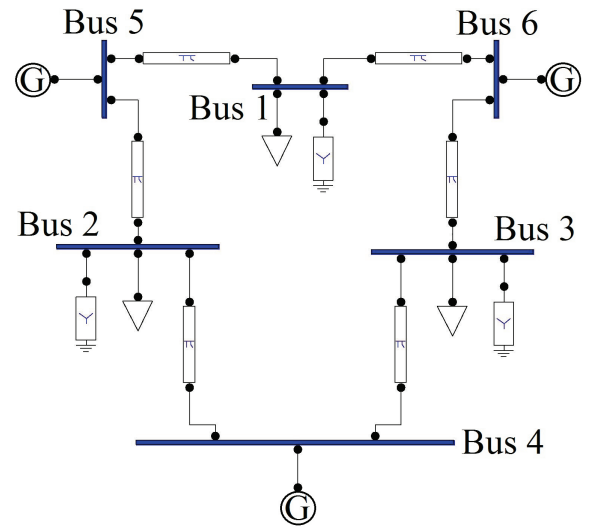

Fig. 1. An example of a power network.

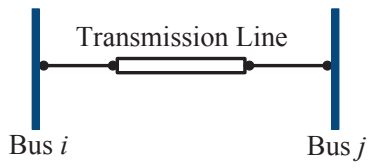

(a)

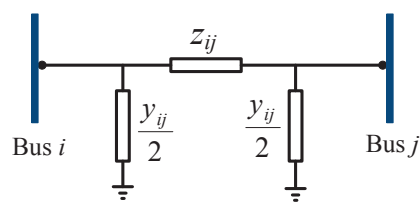

(b)
Fig. 2. (a): A transmission line connecting bus $i$ to bus $j$; (b): Equivalent $\Pi$ model of the transmission line connecting bus $i$ to bus $j$.

to the network topology. Indeed, finding a convex formula relating the power loss to the network configuration has direct applications in two important problems that have been studied in the literature using heuristic algorithms:

- Optimal reconfiguration in distribution networks: The last stage of power delivery is often accomplished by a radial distributed network, such as the one depicted in Figure 3(c), in which all load buses are connected to a feeder (slack bus 1 in the figure) through a tree-shaped network. This network is reconfigurable to some extent in practice; for example, bus 4 in Figure 3(c) can be disconnected from bus 1 and get connected to bus 2 . The goal is to find a configuration whose associated power loss is minimum.

- Optimal placement/sizing of the distributed generation in distribution networks: This problem is concerned with finding the optimal location and power values of distributed generators, mainly based on renewable resources, whose addition to a radial distribution network minimizes the loss.

\section{Power Loss And Network Topology}

To simplify the presentation, assume for now that

- Bus $n$ is the only generator bus, with a prescribed magnitude of voltage equal to $V_{0}$.

- The load bus $k$ is required to deliver the pre-specified power $P_{k}+Q_{k}$ i, for every $k \in\{1,2, \ldots, n-1\}$.

Define $P_{\text {loss }}$ as the minimum of the active power loss that must be incurred in the network so that the above requirements are met. In other words, $P_{\text {loss }}$ is equal to the minimum 
of the total loss

$$
\operatorname{Re}\left\{V_{n} I_{n}^{*}\right\}-\sum_{k=1}^{n-1} P_{k},
$$

subject to the following power, voltage and network constraints:

$$
\begin{aligned}
V_{k} I_{k}^{*} & =-P_{k}-Q_{k} \mathrm{i}, \quad k=1,2, \ldots, n-1, \\
\left|V_{n}\right| & =V_{0}, \\
I & =Y V .
\end{aligned}
$$

The objective is to study how $P_{\text {loss }}$ is related to the topology of the network. The results being developed here will be generalized in Section $\mathrm{V}$ to the multi-generator case with more voltage and power constraints.

Let $e_{1}, e_{2}, \ldots, e_{n}$ denote the standard basis vectors in $\mathbf{R}^{n}$. For every $k=1,2, \ldots, n$, define

$$
\begin{aligned}
Y_{k} & :=e_{k} e_{k}^{*} Y, \\
\mathbf{Y}_{k} & :=\frac{1}{2}\left[\begin{array}{cc}
\operatorname{Re}\left\{Y_{k}+Y_{k}^{T}\right\} & \operatorname{Im}\left\{Y_{k}^{T}-Y_{k}\right\} \\
\operatorname{Im}\left\{Y_{k}-Y_{k}^{T}\right\} & \operatorname{Re}\left\{Y_{k}+Y_{k}^{T}\right\}
\end{array}\right], \\
\overline{\mathbf{Y}}_{k} & :=\frac{-1}{2}\left[\begin{array}{cc}
\operatorname{Im}\left\{Y_{k}+Y_{k}^{T}\right\} & \operatorname{Re}\left\{Y_{k}-Y_{k}^{T}\right\} \\
\operatorname{Re}\left\{Y_{k}^{T}-Y_{k}\right\} & \operatorname{Im}\left\{Y_{k}+Y_{k}^{T}\right\}
\end{array}\right], \\
\mathbf{Y} & :=\left[\begin{array}{cc}
\operatorname{Re}\{Y\} & 0 \\
0 & \operatorname{Re}\{Y\}
\end{array}\right],
\end{aligned}
$$

Define also $M \in \mathbf{R}^{2 n \times 2 n}$ as a diagonal matrix whose entries are all equal to zero, except for its $(n, n)$ and $(2 n, 2 n)$ entries that are equal to 1 .

Optimization 1: Given the variables $\mu \in \mathbf{R}$ and

$$
\begin{aligned}
& \boldsymbol{\lambda}:=\left[\begin{array}{lll}
\lambda_{1} & \cdots & \lambda_{n-1}
\end{array}\right]^{T} \in \mathbf{R}^{n-1}, \\
& \overline{\boldsymbol{\lambda}}:=\left[\begin{array}{lll}
\bar{\lambda}_{1} & \cdots & \bar{\lambda}_{n-1}
\end{array}\right]^{T} \in \mathbf{R}^{n-1},
\end{aligned}
$$

maximize the linear function

$$
f(\boldsymbol{\lambda}, \overline{\boldsymbol{\lambda}}, \mu):=\sum_{k=1}^{n-1}\left(\lambda_{k}-1\right) P_{k}+\sum_{k=1}^{n-1} \bar{\lambda}_{k} Q_{k}-\mu V_{0}^{2},
$$

subject to the linear matrix inequality

$$
\Phi(\boldsymbol{\lambda}, \overline{\boldsymbol{\lambda}}, \mu):=\mathbf{Y}_{n}+\sum_{k=1}^{n-1} \lambda_{k} \mathbf{Y}_{k}+\sum_{k=1}^{n-1} \bar{\lambda}_{k} \overline{\mathbf{Y}}_{k}+\mu M \succeq 0 .
$$

Denote the optimal value of the function (3) with $P_{\min }$.

Theorem 1: The quantity $P_{\min }$ is a nonnegative lower bound on the minimum loss $P_{\text {loss }}$.

Proof: Recall that $P_{\text {loss }}$ can be obtained using the optimization problem specified in (1) and (2). To solve this problem, define the Lagrangian

$$
\begin{aligned}
L(\boldsymbol{\lambda}, \overline{\boldsymbol{\lambda}}, \mu, V): & =\sum_{k=1}^{n-1} \lambda_{k}\left(\operatorname{Re}\left\{V_{k} I_{k}^{*}\right\}+P_{k}\right), \\
& +\sum_{k=1}^{n-1} \bar{\lambda}_{k}\left(\operatorname{Im}\left\{V_{k} I_{k}^{*}\right\}+Q_{k}\right) \\
& +\mu\left(V_{n} V_{n}^{*}-V_{0}^{2}\right)+\operatorname{Re}\left\{V_{n} I_{n}^{*}\right\}-\sum_{k=1}^{n-1} P_{k},
\end{aligned}
$$

where $I=Y V$. The inequality

$$
\max _{\boldsymbol{\lambda}, \overline{\boldsymbol{\lambda}}, \mu} \min _{V} L(\boldsymbol{\lambda}, \overline{\boldsymbol{\lambda}}, \mu, V) \leq P_{\text {loss }}
$$

holds by the weak duality theorem. In order to prove the theorem, it suffices to show that the left side of the above inequality is nonnegative and equal to $P_{\min }$. To this end, notice that

$$
\begin{aligned}
\operatorname{Re}\left\{V_{k} I_{k}^{*}\right\} & =\operatorname{Re}\left\{V^{*} e_{k} e_{k}^{*} I\right\}=\operatorname{Re}\left\{V^{*} Y_{k} V\right\} \\
& =W^{T}\left[\begin{array}{cc}
\operatorname{Re}\left\{Y_{k}\right\} & -\operatorname{Im}\left\{Y_{k}\right\} \\
\operatorname{Im}\left\{Y_{k}\right\} & \operatorname{Re}\left\{Y_{k}\right\}
\end{array}\right] W \\
& =\frac{1}{2} W^{T}\left[\begin{array}{cc}
\operatorname{Re}\left\{Y_{k}+Y_{k}^{T}\right\} & \operatorname{Im}\left\{Y_{k}^{T}-Y_{k}\right\} \\
\operatorname{Im}\left\{Y_{k}-Y_{k}^{T}\right\} & \operatorname{Re}\left\{Y_{k}+Y_{k}^{T}\right\}
\end{array}\right] W \\
& =W^{T} \mathbf{Y}_{k} W,
\end{aligned}
$$

for every $k \in\{1,2, \ldots, n\}$, where

$$
W:=\left[\operatorname{Re}\{V\}^{T} \quad \operatorname{Im}\{V\}^{T}\right]^{T} .
$$

Similarly,

$$
\operatorname{Im}\left\{V_{k} I_{k}^{*}\right\}=W^{T} \overline{\mathbf{Y}}_{k} W
$$

Thus, one can write

$$
L(\boldsymbol{\lambda}, \overline{\boldsymbol{\lambda}}, \mu, V)=f(\boldsymbol{\lambda}, \overline{\boldsymbol{\lambda}}, \mu)+W^{T} \Phi(\boldsymbol{\lambda}, \overline{\boldsymbol{\lambda}}, \mu) W .
$$

By fixing $\lambda, \bar{\lambda}, \mu$, observe that the minimum of the quadratic function $W^{T} \Phi(\boldsymbol{\lambda}, \overline{\boldsymbol{\lambda}}, \mu) W$ with respect to the complexvalued variable $V$ (or the real-valued variable $W$ ), is either 0 or $-\infty$, depending on whether the symmetric matrix $\Phi(\boldsymbol{\lambda}, \overline{\boldsymbol{\lambda}}, \mu)$ is positive semidefinite. This implies that the unconstrained optimization $\max _{\boldsymbol{\lambda}, \overline{\boldsymbol{\lambda}}, \mu} \min _{V} L(\boldsymbol{\lambda}, \overline{\boldsymbol{\lambda}}, \mu, V)$ is tantamount to the maximization of $f(\boldsymbol{\lambda}, \overline{\boldsymbol{\lambda}}, \mu)$ subject to the constraint $\Phi(\boldsymbol{\lambda}, \overline{\boldsymbol{\lambda}}, \mu) \succeq 0$, which is identical to Optimization 1 . Hence, $\max _{\boldsymbol{\lambda}, \overline{\boldsymbol{\lambda}}, \mu} \min _{V} L(\boldsymbol{\lambda}, \overline{\boldsymbol{\lambda}}, \mu, V)=P_{\min }$. Now, it remains to show that $P_{\text {min }} \geq 0$. For this purpose, define $\mathbf{0}_{n-1}$ and $\mathbf{1}_{n-1}$ as the vector of zeros and the vector of ones in $\mathbf{R}^{n-1}$, respectively. The proof is completed by noting that $\left(\mathbf{1}_{n-1}, \mathbf{0}_{n-1}, 0\right)$ is a feasible point of Optimization 1 , where $f\left(\mathbf{1}_{n-1}, \mathbf{0}_{n-1}, 0\right)=0$ (note that the real part of $Y$ is positive semidefinite).

Theorem 1 states that solving Optimization 1 leads to a sensible (nonnegative) lower bound on the active power loss. Two important properties of this optimization problem are as follows:

- Optimization 1 is a semidefinite program and, therefore, its globally optimal solution can be found efficiently [14].

- In the case when the optimal value $P_{\min }$ is equal to $+\infty$, the associated power flow problem must be infeasible (because a lower bound on the power loss is obtained to be infinity). As a result, Optimization 1 is a sanity test for checking the feasibility of a power flow problem.

Remark 1: The objective function $f(\boldsymbol{\lambda}, \overline{\boldsymbol{\lambda}}, \mu)$ in Optimization 1 depends on the load demands as well as the voltage set point. In contrast, the constraint $\Phi(\boldsymbol{\lambda}, \overline{\boldsymbol{\lambda}}, \mu) \succeq 0$ in this optimization problem is contingent upon the physical topology of the distribution network (reflected by $Y$ ). Hence, 
Optimization 1 decomposes the network topology from the load profile. This significant property opens up the possibility of systematically addressing many important problems, such as (near) optimal network reconfiguration design.

Remark 2: Since Theorem 1 is based on applying the Lagrangian theory to a nonlinear optimization problem, strong duality may not hold. As a result, $P_{\text {min }}$ might not be equal to $P_{\text {loss }}$. In the case when there exists no duality gap, the optimal bus voltages can be recovered from Optimization 1 . More precisely, the vector $\left[\operatorname{Re}\left\{V^{\text {opt }}\right\}^{T} \quad \operatorname{Im}\left\{V^{\text {opt }}\right\}^{T}\right]^{T}$ is an eigenvector of the singular matrix $\Phi\left(\boldsymbol{\lambda}^{\mathrm{opt}}, \overline{\boldsymbol{\lambda}}^{\mathrm{opt}}, \mu^{\mathrm{opt}}\right)$ associated with its zero eigenvalue, where $\left(\boldsymbol{\lambda}^{\mathrm{opt}}, \overline{\boldsymbol{\lambda}}^{\mathrm{opt}}, \mu^{\mathrm{opt}}\right)$ is a maximizer of Optimization 1.

Remark 3: Recall that $\sum_{k=1}^{n-1}\left(\lambda_{k}^{\text {opt }}-1\right) P_{k}+$ $\sum_{k=1}^{n-1} \bar{\lambda}_{k}^{\text {opt }} Q_{k}-\mu^{\text {opt }} V_{0}^{2}$ is either equal to or possibly a lower bound on $P_{\text {loss. }}$. Now, assume that the goal is to identify a load bus incurring the most power loss so that a small generator is installed to compensate for its active power consumption. The above lower bound suggests choosing a bus $k$ whose Lagrange multiplier $\lambda_{k}^{\text {opt }}$ has the highest value (because any deduction in $P_{k}$ is amplified by the factor $\left.\lambda_{k}^{\mathrm{opt}}-1\right)$. In other words, the Lagrange multipliers $\lambda_{1}^{\text {opt }}, \ldots, \lambda_{n-1}^{\text {opt }}$ roughly determine the contributions of different buses on the power loss. The validity of this interpretation will be later verified in simulations. This idea can be generalized to tackle the problem of sizing/placement of distributed generation.

Although $P_{\min }$ is only a lower bound on $P_{\text {loss }}$, we have verified that the equality $P_{\text {loss }}=P_{\text {min }}$ holds for all IEEE test systems with 14, 30, 57, 118 and 300 buses as well as numerous other power systems. This observation implies that power systems have some hidden structures, which could bridge the duality gap. In what follows, we first derive a condition under which $P_{\text {loss }}=P_{\min }$ holds and then explain why this condition is likely to be satisfied in practice.

\section{DuAlity GAP}

The proof of Theorem 1 yields that $P_{\text {loss }}$ can be obtained by minimizing

$$
W^{T} \mathbf{Y}_{n} W-\sum_{k=1}^{n-1} P_{k}
$$

over the real-valued variable $W \in \mathbf{R}^{2 n}$ subject to

$$
\begin{aligned}
W^{T} \mathbf{Y}_{k} W & =-P_{k}, \quad k=1,2, \ldots, n-1 \\
W^{T} \overline{\mathbf{Y}}_{k} W & =-Q_{k}, \quad k=1,2, \ldots, n-1, \\
W^{T} M W & =V_{0}^{2} .
\end{aligned}
$$

If $X$ is defined as $W W^{T}$, the above optimization will be tantamount to the minimization of

$$
\operatorname{trace}\left\{\mathbf{Y}_{n} X\right\}-\sum_{k=1}^{n-1} P_{k}
$$

subject to

$$
\begin{aligned}
\operatorname{trace}\left\{\mathbf{Y}_{k} X\right\} & =-P_{k}, \quad k=1,2, \ldots, n-1, \\
\operatorname{trace}\left\{\overline{\mathbf{Y}}_{k} X\right\} & =-Q_{k}, \quad k=1,2, \ldots, n-1, \\
\operatorname{trace}\{M X\} & =V_{0}^{2}, \\
X & \succeq 0, \\
\operatorname{rank}(X) & =1 .
\end{aligned}
$$

The non-convexity of this optimization problem arises from the constraint $\operatorname{rank}(X)=1$.

Lemma 1: The dual of the nonconvex problem of the power loss minimization, i.e. Optimization 1, is the same as the dual of the convex optimization derived from (6) and (7) by removing the rank constraint. Moreover, $X$ in the latter optimization plays the role of the Lagrange multiplier for the matrix constraint of Optimization 1.

Proof: The proof is omitted due to space restrictions and may be found in [15].

For simplicity in the proof and with no loss of generality, assume that $P_{\text {loss }}$ is finite and that the feasibility region of Optimization 1 has a non-empty interior. These assumptions are made to ensure that strong duality holds between Optimization 1 and its dual.

Theorem 2: Assume that $\Phi\left(\boldsymbol{\lambda}^{\mathrm{opt}}, \overline{\boldsymbol{\lambda}}^{\mathrm{opt}}, \mu^{\mathrm{opt}}\right)$ has rank greater than or equal to $2 n-2$. The lower bound $P_{\min }$ obtained from Optimization 1 is the same as the minimum power loss $P_{\text {loss }}$.

Sketch of Proof: Consider the problem of minimizing

$$
\operatorname{trace}\{\mathbf{Y} X\}-\sum_{k=1}^{n-1} P_{k}
$$

subject to

$$
\begin{aligned}
\operatorname{trace}\left\{\mathbf{Y}_{k} X\right\} & =-P_{k}, \quad k=1,2, \ldots, n-1, \\
\operatorname{trace}\left\{\overline{\mathbf{Y}}_{k} X\right\} & =-Q_{k}, \quad k=1,2, \ldots, n-1, \\
\operatorname{trace}\{M X\} & =V_{0}^{2}, \\
X & \succeq 0 .
\end{aligned}
$$

Following the discussion made before Lemma 1, it suffices to show that the above optimization problem has a rank-one solution $X$. To this end, let $X^{\text {opt }}$ be an arbitrary minimizer of this problem. It follows from the complementary slackness conditions and Lemma 1 that

$$
\begin{aligned}
& \Phi\left(\boldsymbol{\lambda}^{\mathrm{opt}}, \overline{\boldsymbol{\lambda}}^{\mathrm{opt}}, \mu^{\mathrm{opt}}\right) \succeq 0, \quad X^{\mathrm{opt}} \succeq 0, \\
& \operatorname{trace}\left\{\Phi\left(\boldsymbol{\lambda}^{\mathrm{opt}}, \overline{\boldsymbol{\lambda}}^{\mathrm{opt}}, \mu^{\mathrm{opt}}\right) X^{\mathrm{opt}}\right\}=0 .
\end{aligned}
$$

Since $\Phi\left(\boldsymbol{\lambda}^{\mathrm{opt}}, \overline{\boldsymbol{\lambda}}^{\mathrm{opt}}, \mu^{\mathrm{opt}}\right)$ has rank at least $2 n-2$, it can be concluded that $X^{\text {opt }}$ has rank at most 2 . If the rank of $X^{\text {opt }}$ is 1 , the proof is complete. Thus, assume that $X_{\text {opt }}$ has rank 2 . Let $\left[\begin{array}{ll}U_{1}^{T} & U_{2}^{T}\end{array}\right]^{T}$ be a nonzero vector in the null space of $\Phi\left(\boldsymbol{\lambda}^{\mathrm{opt}}, \overline{\boldsymbol{\lambda}}^{\mathrm{opt}}, \mu^{\mathrm{opt}}\right)$, for some real vectors $U_{1}, U_{2} \in \mathbf{R}^{n}$. It is easy to verify that $\left[\begin{array}{cc}U_{2}^{T} & -U_{1}^{T}\end{array}\right]^{T}$ is also in the null space of $\Phi\left(\lambda^{\text {opt }}, \bar{\lambda}^{\text {opt }}, \mu^{\text {opt }}\right)$. The relations given in (10) yield that 
there must be two positive scalars $\alpha$ and $\beta$ such that

$$
\begin{aligned}
X^{\mathrm{opt}} & =\alpha\left[\begin{array}{c}
U_{1} \\
U_{2}
\end{array}\right]\left[\begin{array}{ll}
U_{1}^{T} & U_{2}^{T}
\end{array}\right] \\
& +\beta\left[\begin{array}{c}
U_{2} \\
-U_{1}
\end{array}\right]\left[\begin{array}{ll}
U_{2}^{T} & -U_{1}^{T}
\end{array}\right] .
\end{aligned}
$$

Now, it can be shown that the rank-one matrix

$$
(\alpha+\beta)\left[\begin{array}{l}
U_{1} \\
U_{2}
\end{array}\right]\left[\begin{array}{ll}
U_{1}^{T} & U_{2}^{T}
\end{array}\right]
$$

is also a minimizer of the optimization problem specified by (8) and (9). This completes the proof.

The relation $\operatorname{rank}\left\{\Phi\left(\mathbf{1}_{n-1}, \mathbf{0}_{n-1}, 0\right)\right\} \geq 2 n-2$ holds due to the power network being strongly connected. This means that the rank of $\Phi(\boldsymbol{\lambda}, \bar{\lambda}, \mu)$ is generically at least $2 n-2$. Therefore, Theorem 2 requires that the condition $\operatorname{rank}\{\Phi(\boldsymbol{\lambda}, \overline{\boldsymbol{\lambda}}, \mu)\} \geq 2 n-2$ that holds generically be satisfied at the optimal point.

\section{A. Zero Duality Gap for Resistive Networks}

The active power loss $P_{\text {loss }}$ is a consequence of the resistive part of the network. In other words, the minimum power loss is zero for a purely inductive/capacitive power network, provided the power flow problem is feasible. In this subsection, we investigate an abstract, nonetheless important, case to gain insight into the zero-duality-gap condition given in Theorem 2. The general case will be discussed in Section V.

Throughout this subsection, assume that the power system is resistive (i.e. $\operatorname{Im}\{Y\}=0$ ) and that there is no impedanceto-ground (i.e. $y_{1}=\cdots=y_{n}=0$ ). A practical power network is normally associated with the well-known property that its required minimum loss reduces if its load demand decreases (due to a reduction in the line currents). In other words, it is likely that replacing the constraints given in (9a) with

$$
\operatorname{trace}\left\{\mathbf{Y}_{k} X\right\} \leq-P_{k}, \quad k=1,2, \ldots, n-1
$$

again leads to the same solution $P_{\min }$. Under this circumstance, the optimal Lagrange multipliers $\lambda_{1}^{\mathrm{opt}}, \ldots, \lambda_{n-1}^{\mathrm{opt}}$ cannot be negative. This property will be rigorously proved in the sequel for a resistive network.

Lemma 2: The Lagrange multipliers $\lambda_{1}^{\mathrm{opt}}, \ldots, \lambda_{n-1}^{\mathrm{opt}}$ are all nonnegative.

Proof: Since the network is resistive, the term $\operatorname{Im}\{Y\}$ and its associated multiplier $\bar{\lambda}$ can be removed from Optimization 1 . Hence, one can write:

$$
\begin{aligned}
& \Phi\left(\boldsymbol{\lambda}^{\mathrm{opt}}, \overline{\boldsymbol{\lambda}}^{\mathrm{opt}}, \mu^{\mathrm{opt}}\right)=\mathbf{Y}_{n}+\sum_{k=1}^{n-1} \lambda_{k}^{\mathrm{opt}} \mathbf{Y}_{k}+\mu^{\mathrm{opt}} M \\
& =D^{\mathrm{opt}}\left(\frac{\mathbf{Y}}{2}+\frac{\mu^{\mathrm{opt}} M}{2}\right)+\left(\frac{\mathbf{Y}}{2}+\frac{\mu^{\mathrm{opt}} M}{2}\right) D^{\mathrm{opt}},
\end{aligned}
$$

where $D^{\text {opt }} \in \mathbf{R}^{2 n \times 2 n}$ is a diagonal matrix with the diagonal entries $\lambda_{1}^{\mathrm{opt}}, \ldots, \lambda_{n-1}^{\mathrm{opt}}, 1, \lambda_{1}^{\mathrm{opt}}, \ldots, \lambda_{n-1}^{\mathrm{opt}}, 1$. Define $\mathbf{1}_{n}$ as the vector of ones in $\mathbf{R}^{n}$. Since there is no impedance-to-ground in the network, the relation $Y \mathbf{1}_{n}=0$ holds. Now, it follows from the positive semi-definiteness of $\Phi\left(\boldsymbol{\lambda}^{\mathrm{opt}}, \overline{\boldsymbol{\lambda}}^{\mathrm{opt}}, \mu^{\mathrm{opt}}\right)$ and (12) that

$$
0 \leq\left[\begin{array}{cc}
\mathbf{1}_{n}^{T} & \mathbf{1}_{n}^{T}
\end{array}\right] \Phi\left(\boldsymbol{\lambda}^{\mathrm{opt}}, \overline{\boldsymbol{\lambda}}^{\mathrm{opt}}, \mu^{\mathrm{opt}}\right)\left[\begin{array}{cc}
\mathbf{1}_{n}^{T} & \mathbf{1}_{n}^{T}
\end{array}\right]^{T}=2 \mu^{\mathrm{opt}},
$$

which implies that $\mu^{\text {opt }}$ is nonnegative. As the first case, assume that $\mu^{\mathrm{opt}}>0$, which makes the matrix $\frac{\mathbf{Y}}{2}+\frac{\mu^{\mathrm{opt}} M}{2}$ positive definite. Using this property, it can be shown that

$$
D^{\mathrm{opt}}=\int_{0}^{\infty} e^{-\left(\frac{\mathrm{Y}}{2}+\frac{\mu^{\mathrm{opt}} M}{2}\right) t} \Phi e^{-\left(\frac{\mathrm{Y}}{2}+\frac{\mu^{\mathrm{opt}} M}{2}\right) t} d t
$$

where $\Phi$ stands for $\Phi\left(\boldsymbol{\lambda}^{\mathrm{opt}}, \overline{\boldsymbol{\lambda}}^{\mathrm{opt}}, \mu^{\mathrm{opt}}\right)$. The above relation simply shows that $D^{\text {opt }}$ is nonnegative and so are its entries $\lambda_{1}^{\mathrm{opt}}, \ldots, \lambda_{n-1}^{\mathrm{opt}}$. As the second case, let $\mu^{\mathrm{opt}}$ be zero. The only extra part in the proof for this case is the necessity to show that the right side of (13) does not become infinity. This follows from the fact that $\mathbf{Y}$ has two orthogonal eigenvectors $\left[\begin{array}{cc}\mathbf{1}_{n}^{T} & \mathbf{1}_{n}^{T}\end{array}\right]^{T}$ and $\left[\begin{array}{cc}\mathbf{1}_{n}^{T} & -\mathbf{1}_{n}^{T}\end{array}\right]^{T}$ corresponding to its zero eigenvalue, which are both in the null space of $\Phi$.

Lemma 2 states that the Lagrange multipliers $\lambda_{1}^{\text {opt }}, \ldots, \lambda_{n-1}^{\text {opt }}$ are all nonnegative. The next theorem shows that the duality gap is zero under a slightly stronger condition of the strict positivity of these multipliers.

Theorem 3: Assume that the Lagrange multipliers $\lambda_{1}^{\text {opt }}, \ldots, \lambda_{n-1}^{\text {opt }}$ are strictly positive. Then, the zero-dualitygap condition given in Theorem 2 holds and therefore $P_{\text {loss }}=P_{\text {min }}$.

Proof: The key to the proof is the following two important properties:

- The matrix $Y$ is irreducible (due to the power network being strongly connected).

- The off-diagonal entries of $Y$ are all non-positive (due to the definition of $Y$ and the positivity of every transmission line's resistance).

On applying the above properties to (12) and using the positivity of $\lambda_{1}^{\mathrm{opt}}, \ldots, \lambda_{n-1}^{\mathrm{opt}}$, one can deduce that $\Phi\left(\boldsymbol{\lambda}^{\mathrm{opt}}, \overline{\boldsymbol{\lambda}}^{\mathrm{opt}}, \mu^{\mathrm{opt}}\right)$ is expressible as

$$
\Phi\left(\boldsymbol{\lambda}^{\mathrm{opt}}, \overline{\boldsymbol{\lambda}}^{\mathrm{opt}}, \mu^{\mathrm{opt}}\right)=\left[\begin{array}{cc}
T & 0 \\
0 & T
\end{array}\right],
$$

where $T$ is an $n \times n$ irreducible matrix with non-positive offdiagonal entries. Now, it follows from the Perron-Frobenius theorem that the multiplicity of the smallest eigenvalue of $\Phi\left(\boldsymbol{\lambda}^{\mathrm{opt}}, \overline{\boldsymbol{\lambda}}^{\mathrm{opt}}, \mu^{\mathrm{opt}}\right)$ is at most 2. Since $\Phi\left(\boldsymbol{\lambda}^{\mathrm{opt}}, \overline{\boldsymbol{\lambda}}^{\mathrm{opt}}, \mu^{\mathrm{opt}}\right)$ is positive semidefinite, this result implies that the rank of $\Phi\left(\boldsymbol{\lambda}^{\mathrm{opt}}, \overline{\boldsymbol{\lambda}}^{\mathrm{opt}}, \mu^{\mathrm{opt}}\right)$ is at least $2 n-2$. This completes the proof.

As can be inferred from Theorem 3, the main reason behind the convexification of the nonconvex problem of loss minimization is the physical property of the network, i.e. the positivity of resistance, which has made the matrix $Y$ have a nice "sign" structure.

The above result can be extended to a general power network. Indeed, we provided two different approaches in [15] to justify that the relation $P_{\text {loss }}=P_{\min }$ is expected to widely hold in practice due to the physical properties of a power network. The details are omitted here for brevity. 


\section{B. Connection to the Rank Minimization Literature}

Combining the constraint (5a) with the objective function (4) leads to the conclusion that $P_{\text {loss }}$ is equal to the minimum of

$$
W^{T} \mathbf{Y} W
$$

subject to

$$
\begin{aligned}
W^{T} \mathbf{Y}_{k} W & =-P_{k}, \quad k=1,2, \ldots, n-1, \\
W^{T} \overline{\mathbf{Y}}_{k} W & =-Q_{k}, \quad k=1,2, \ldots, n-1, \\
W^{T} M W & =V_{0}^{2} .
\end{aligned}
$$

Assume, for simplicity, that $\operatorname{det}\{\mathbf{Y}\} \neq 0$. Since $\mathbf{Y}$ is positive definite, its square root exists. Let $\mathbf{Y}^{\frac{1}{2}}$ denote the unique, symmetric, positive definite matrix whose square is equal to Y. Define $\tilde{X}$ as

$$
\tilde{X}:=\mathbf{Y}^{\frac{1}{2}} W W^{T} \mathbf{Y}^{\frac{1}{2}} .
$$

Notice that

$$
W^{T} \mathbf{Y} W=\operatorname{trace}\left\{W^{T} \mathbf{Y} W\right\}=\operatorname{trace}\{\tilde{X}\}
$$

It follows from (14) and (15) that $P_{\text {loss }}$ is equal to the minimum of the function

$$
\|\tilde{X}\|_{*}
$$

subject to

$$
\begin{aligned}
\operatorname{trace}\left\{\mathbf{Y}^{-\frac{1}{2}} \mathbf{Y}_{k} \mathbf{Y}^{-\frac{1}{2}} \tilde{X}\right\} & =-P_{k}, k=1,2, \ldots, n-1, \\
\operatorname{trace}\left\{\mathbf{Y}^{-\frac{1}{2}} \overline{\mathbf{Y}}_{k} \mathbf{Y}^{-\frac{1}{2}} \tilde{X}\right\} & =-Q_{k}, k=1,2, \ldots, n-1, \\
\operatorname{trace}\left\{\mathbf{Y}^{-\frac{1}{2}} M \mathbf{Y}^{-\frac{1}{2}} \tilde{X}\right\} & =V_{0}^{2}, \\
\tilde{X} & \succ 0, \\
\operatorname{rank}(\tilde{X}) & =1 .
\end{aligned}
$$

The rank constraint (16e) makes this optimization problem nonconvex, in general. On the other hand, it is shown in [16] and [17] that a good heuristic for solving a rank-constraint feasibility problem is to replace the constraint $\operatorname{rank}(\tilde{X})=1$ with an objective function min $\|\tilde{X}\|_{*}$. Interestingly, the above optimization problem automatically minimizes the norm of $\tilde{X}$. In other words, minimizing the loss also minimizes the nuclear norm of the variable $\tilde{X}$, which in turn would reduce the rank of $\tilde{X}$. This important property of a loss minimization problem could be another reason behind the satisfaction of the relation $P_{\text {loss }}=P_{\min }$ widely in practice (note that $P_{\min }$ is obtained from the dual of the above optimization problem after removing its rank constraint).

\section{Generalization}

Consider a power network with $n$ buses, labeled as $1, \ldots, n$, where all buses are possibly directly connected to loads, but only the first $m$ buses are directly connected to generators. For $k \in\{1, \ldots, n\}$ and $l \in\{1, \ldots, m\}$, define the following quantities:

- $P_{k}^{d}$ and $Q_{k}^{d}$ : Known active and reactive loads at bus $k$, respectively.

- $P_{l}^{g}$ and $Q_{l}^{g}$ : Unknown active and reactive powers generated at bus $l$, respectively.
- $V_{k}$ : Unknown complex voltage at bus $k$.

Instead of the loss minimization problem, we equivalently consider the problem of the total generation minimization, which aims to minimize

$$
\sum_{l=1}^{m} P_{G_{l}}
$$

over the parameters $V_{1}, \ldots, V_{n}, P_{G_{1}}, \ldots, P_{G_{m}}, Q_{G_{1}}, \ldots, Q_{G_{m}}$, subject to the constraints

$$
\begin{array}{r}
P_{l}^{\min } \leq P_{G_{l}} \leq P_{l}^{\max }, l=1,2, \ldots, m, \\
Q_{l}^{\min } \leq Q_{G_{l}} \leq Q_{l}^{\max }, l=1,2, \ldots, m, \\
V_{k}^{\min } \leq\left|V_{k}\right| \leq V_{k}^{\max }, k=1,2, \ldots, n, \\
V_{l} I_{l}^{*}=\left(P_{G_{l}}-P_{D_{l}}\right)+\left(Q_{G_{l}}-Q_{D_{l}}\right) \mathrm{i}, l=1,2, \ldots, m, \\
V_{k} I_{k}^{*}=-P_{D_{k}}-Q_{D_{k}} \mathrm{i}, k=m+1, \ldots, n .
\end{array}
$$

In this problem, the first three inequalities limit the power and voltage parameters by the given bounds $P_{l}^{\min }, P_{l}^{\max }, Q_{l}^{\min }, Q_{l}^{\max }, V_{k}^{\min }, V_{k}^{\max }$, whereas the last two equations express the constraints imposed by the network. There could be more constraints, e.g. line flow limits, which can be easily incorporated. Note that the loss minimization problem is generally hard and NP-complete in the worst-case [15].

Extend the definition of $P_{k}^{\min }, P_{k}^{\max }, Q_{k}^{\min }, Q_{k}^{\max }$ to $k \in$ $\{m+1, \ldots, n\}$, with $P_{k}^{\min }=P_{k}^{\max }=Q_{k}^{\min }=Q_{k}^{\max }=0$ if $k \in\{m+1, \ldots, n\}$. For every $l=1,2, \ldots, n$, define also $M_{l} \in \mathbf{R}^{2 n \times 2 n}$ as a diagonal matrix whose entries are all equal to zero, except for its $(l, l)$ and $(n+l, n+l)$ entries that are equal to 1 . The following optimization is the general form of Optimization 1.

Optimization 2: Maximize the function

$$
\begin{aligned}
& f(\boldsymbol{\lambda}, \overline{\boldsymbol{\lambda}}, \boldsymbol{\mu}):=\sum_{k=1}^{n}\left\{\lambda_{k}^{\min } P_{k}^{\min }-\lambda_{k}^{\max } P_{k}^{\max }+\boldsymbol{\lambda}_{k} P_{D_{k}}\right. \\
& +\bar{\lambda}_{k}^{\min } Q_{k}^{\min }-\bar{\lambda}_{k}^{\max } Q_{k}^{\max }+\overline{\boldsymbol{\lambda}}_{k} Q_{D_{k}}+\mu_{k}^{\min }\left(V_{k}^{\min }\right)^{2} \\
& \left.-\mu_{k}^{\max }\left(V_{k}^{\max }\right)^{2}\right\}
\end{aligned}
$$

over the nonnegative scalar variables $\lambda_{k}^{\min }, \lambda_{k}^{\max }$, $\bar{\lambda}_{k}^{\min }, \bar{\lambda}_{k}^{\max }, \mu_{k}^{\min }, \mu_{k}^{\max }, k=1,2, \ldots, n$, subject to

$$
\Phi(\boldsymbol{\lambda}, \overline{\boldsymbol{\lambda}}, \boldsymbol{\mu}):=\sum_{k=1}^{n}\left(\boldsymbol{\lambda}_{k} \mathbf{Y}_{k}+\overline{\boldsymbol{\lambda}}_{k} \overline{\mathbf{Y}}_{k}+\boldsymbol{\mu}_{k} M_{k}\right) \succeq 0,
$$

where

$$
\begin{aligned}
\boldsymbol{\lambda}_{k} & :=\left\{\begin{array}{cc}
-\lambda_{k}^{\min }+\lambda_{k}^{\max }+1 & \text { if } k=1, \ldots, m \\
-\lambda_{k}^{\min }+\lambda_{k}^{\max } & \text { otherwise }
\end{array}\right. \\
\overline{\boldsymbol{\lambda}}_{k} & :=-\bar{\lambda}_{k}^{\min }+\bar{\lambda}_{k}^{\max } \\
\boldsymbol{\mu}_{k} & :=-\mu_{k}^{\min }+\mu_{k}^{\max },
\end{aligned}
$$

for every $k \in\{1,2, \ldots, n\}$.

Note that $\boldsymbol{\lambda}, \overline{\boldsymbol{\lambda}}$ and $\boldsymbol{\mu}$ in Optimization 2 are the vectors associated with the sets $\left\{\lambda_{k}^{\min }, \lambda_{k}^{\max }\right\}_{k=1}^{n},\left\{\bar{\lambda}_{k}^{\min }, \bar{\lambda}_{k}^{\max }\right\}_{k=1}^{n}$ and $\left\{\mu_{k}^{\min }, \mu_{k}^{\max }\right\}_{k=1}^{n}$, respectively.

Theorem 4: Assume that there exists a solution $\left(\boldsymbol{\lambda}^{\mathrm{opt}}, \overline{\boldsymbol{\lambda}}^{\mathrm{opt}}, \boldsymbol{\mu}^{\mathrm{opt}}\right)$ to Optimization 2 such that $\Phi\left(\boldsymbol{\lambda}^{\mathrm{opt}}, \overline{\boldsymbol{\lambda}}^{\mathrm{opt}}\right.$, 
$\left.\boldsymbol{\mu}^{\text {opt }}\right)$ has rank greater than or equal to $2 n-2$. The optimal objective value of Optimization 2 is identical to the minimum value of the total generation. Moreover, the optimal vector of bus voltages satisfies the relation

$$
V^{\mathrm{opt}}=\left(\zeta_{1}+\zeta_{2} \mathrm{i}\right)\left(U_{1}+U_{2} \mathrm{i}\right)
$$

for some real numbers $\zeta_{1}, \zeta_{2}$ and a vector $\left[\begin{array}{cc}U_{1}^{T} & U_{2}^{T}\end{array}\right]^{T}$ in the null space of $\Phi\left(\boldsymbol{\lambda}^{\mathrm{opt}}, \overline{\boldsymbol{\lambda}}^{\mathrm{opt}}, \boldsymbol{\mu}^{\mathrm{opt}}\right)$.

Theorem 4 can be proved in line with the proof of Theorem 2. As we have studied in [15], the rank condition given in Theorem 4 is likely to hold widely in practice due to the two properties associated with power systems (see the proof of Theorem 3): (i) the positivity of the Lagrange multipliers $\lambda_{1}^{\mathrm{opt}}, \ldots, \boldsymbol{\lambda}_{n}^{\mathrm{opt}}$, (ii) the particular sign structures of the real and imaginary parts of $Y$.

\section{Simulation Results}

Our method can be exploited to verify that the duality gap is zero for all IEEE systems with 14, 30, 57, 118 and 300 buses, where the goal is to minimize either the total generation cost or the power loss. The details of this interesting observation can be found in [15]. Since these systems are so large that the specific values of the optimal solutions cannot be provided here, some smaller examples will be analyzed in the sequel instead.

Consider the three systems depicted in Figure 3, which are referred to as Systems 1, 2 and 3. In each of these systems, bus 1 is the slack bus (feeder) whose voltage is set to a specific value and other buses are the load buses. The detailed specifications of these systems (together with the load demands) are provided in Table $\mathrm{I}$ in per unit for the voltage rating $400 \mathrm{kV}$ and the power rating 100MVA. Optimization 1 is solved for each system and the corresponding Lagrange multipliers are summarized in Table II. As expected from Lemma 2, it can be seen that $\lambda_{2}^{\mathrm{opt}}, \lambda_{3}^{\mathrm{opt}}, \lambda_{4}^{\mathrm{opt}}$ are all nonnegative. Interestingly, the zero-duality-gap condition given in Theorem 2 is satisfied for all these systems. The recovered optimal voltages and power losses are brought in Table III.

We repeated this example several hundred times by randomly choosing the parameters of these systems over a wide range of values. In all these trials, Optimization 1 always found $P_{\text {loss }}$ or detected the infeasibility of the corresponding power flow problem. We also validated our simulations using the toolbox PSAT [18]. Another advantage of Optimization 1 is in proving that a specific power flow problem is infeasible. For instance, if the voltage magnitude at the slack bus of System 1 is changed from 1.05 to 1 , Optimization 1 becomes unbounded. Hence, the power loss in this case becomes infinity, which is an indication of the fact that the corresponding power flow problem is infeasible.

Optimization 1 also provides valuable insights into the connection between the power loss and the network topology. For example, consider System 3 and assume that any arbitrary amount of reactive power can be injected at every load bus. It follows from a variant of Optimization 1 that the active power loss can be reduced from 0.3877 to 0.3542
TABLE I

PARAMETERS OF THE SYSTEMS GIVEN IN FigURE 3.

\begin{tabular}{|c|c|c|c|}
\hline Parameters & System 1 & System 2 & System 3 \\
\hline$z_{12}$ & $0.05+0.25 \mathrm{i}$ & $0.1+0.5 \mathrm{i}$ & $0.10+0.1 \mathrm{i}$ \\
\hline$z_{13}$ & $0.04+0.40 \mathrm{i}$ & None & None \\
\hline$z_{23}$ & $0.02+0.10 \mathrm{i}$ & $0.02+0.20 \mathrm{i}$ & $0.01+0.1 \mathrm{i}$ \\
\hline$z_{14}$ & None & None & $0.01+0.2 \mathrm{i}$ \\
\hline$y_{12}$ & $0.06 \mathrm{i}$ & $0.02 \mathrm{i}$ & $0.06 \mathrm{i}$ \\
\hline$y_{13}$ & $0.05 \mathrm{i}$ & None & None \\
\hline$y_{23}$ & $0.02 \mathrm{i}$ & $0.02 \mathrm{i}$ & $0.02 \mathrm{i}$ \\
\hline$y_{14}$ & None & None & $0.02 \mathrm{i}$ \\
\hline$V_{0}$ & 1.05 & 1.4 & 1 \\
\hline$P_{2}+Q_{2} \mathrm{i}$ & $0.95+0.4 \mathrm{i}$ & $0.7+0.02 \mathrm{i}$ & $0.9+0.02 \mathrm{i}$ \\
\hline$P_{3}+Q_{3} \mathrm{i}$ & $0.9+0.6 \mathrm{i}$ & $0.65+0.02 \mathrm{i}$ & $0.6+0.02 \mathrm{i}$ \\
\hline$P_{4}+Q_{4} \mathrm{i}$ & None & None & $0.9+0.02 \mathrm{i}$ \\
\hline
\end{tabular}

TABLE II

LAGRANGE MULTIPLIERS OBTAINED BY SOLVING OPTIMIZATION 1 FOR THE SYSTEMS GIVEN IN FIGURE 3.

\begin{tabular}{|c|c|c|c|}
\hline Lagrange Multipliers & System 1 & System 2 & System 3 \\
\hline$\lambda_{2}^{\text {opt }}$ & 1.3809 & 1.4028 & 1.7176 \\
\hline$\lambda_{3}^{\text {opt }}$ & 1.4155 & 1.4917 & 1.7900 \\
\hline$\lambda_{4}^{\text {opt }}$ & None & None & 1.0207 \\
\hline $\bar{\lambda}_{2}^{\text {opt }}$ & 0.4391 & 0.2508 & 0.1764 \\
\hline $\bar{\lambda}_{3}^{\text {opt }}$ & 0.4955 & 0.2633 & 0.1858 \\
\hline $\bar{\lambda}_{4}^{\text {opt }}$ & None & None & 0.0061 \\
\hline$\mu^{\text {opt }}$ & 0.0005 & 0.0001 & 0.0005 \\
\hline
\end{tabular}

(per unit) under this circumstance. Since this improvement is not significant, it is desired to further reduce the active power loss by installing a small active source (generator) at one of buses 2,3 or 4 with the capacity of 0.3 . For this purpose, notice that the Lagrange multipliers associated with the power loss 0.3542 are $\lambda_{2}^{\text {opt }}=1.5874, \lambda_{3}^{\text {opt }}=$ 1.6153 , and $\lambda_{4}^{\mathrm{opt}}=1.0186$. These numbers indicate that the order of buses in causing the power loss is $3,2,4$ (see Remark 3). Hence, this optimization suggests that the small generator be installed at bus 3. Indeed, it can be verified that the power losses associated with installing the generator at buses $3,2,4$ are $0.2043,0.2092,0.3496$, respectively. Hence, the Lagrange multipliers correctly identified the bus whose power compensation had the greatest effect on power loss.

\section{CONCLUSIONS}

Given a power system with certain constraints on its physical variables, this paper proposes a linear-matrix-inequality

TABLE III

PARAMETERS RECOVERED FROM OPTIMIZATION 1 FOR THE SYSTEMS GIVEN IN FIGURE 3.

\begin{tabular}{|c|c|c|c|}
\hline $\begin{array}{l}\text { Recovered } \\
\text { Parameters }\end{array}$ & System 1 & System 2 & System 3 \\
\hline$V_{1}^{\text {opt }}$ & $1.05 \angle 0^{\circ}$ & $1.4 \angle 0^{\circ}$ & $1 \angle 0^{\circ}$ \\
\hline$V_{2}^{\text {opt }}$ & $0.71 \angle-20.11^{\circ}$ & $1.10 \angle-25.73^{\circ}$ & $0.78 \angle-10.58^{\circ}$ \\
\hline$V_{3}^{\text {opt }}$ & $0.68 \angle-21.94^{\circ}$ & $1.08 \angle-31.96^{\circ}$ & $0.76 \angle-16.31^{\circ}$ \\
\hline$V_{4}^{\text {opt }}$ & None & None & $0.95 \angle-10.82^{\circ}$ \\
\hline$P_{\text {loss }}$ & 0.2193 & 0.1588 & 0.3877 \\
\hline
\end{tabular}




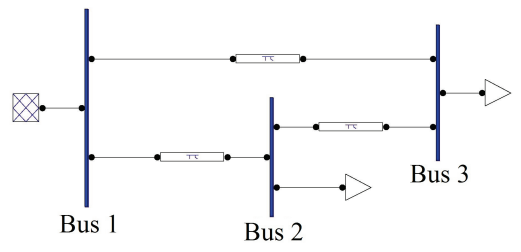

(a)

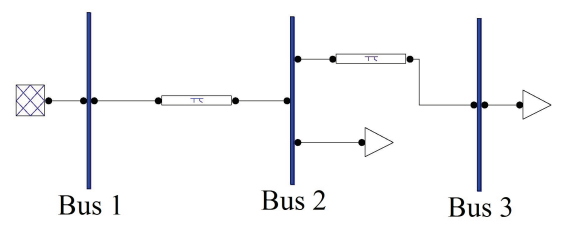

(b)

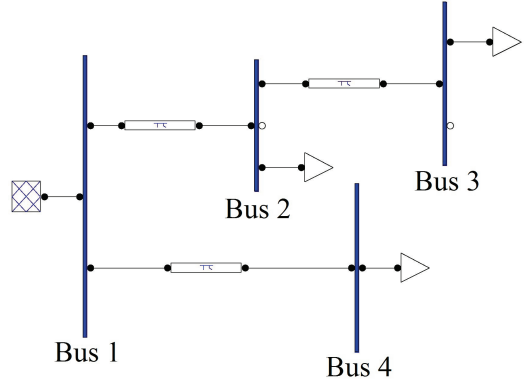

(c)

Fig. 3. Figures (a), (b) and (c) depict Systems 1, 2 and 3 studied in Section VI, respectively.

(LMI) optimization problem to find a lower bound on the minimum amount of active (reactive) power loss incurred in the network. This LMI problem has the useful property that its objective function depends on the load profile and its matrix inequality constraint is contingent only upon the topology of the network. This implies that the network topology determines the shape of the feasibility region within which the Lagrange multipliers that minimize the power loss must lie. Direct applications of this result are in optimal network reconfiguration and optimal placement/sizing of distributed generation units in distribution networks. It is also observed in many examples, including IEEE benchmark systems with 14, 30, 57, 118 and 300 buses, that the proposed LMI optimization problem always generates the exact value of the power loss, as opposed to a lower bound on it. As justified mathematically, this result is expected to hold widely in practice.

\section{ACKNOWLEDGMENTS}

The authors are thankful to Prof. John C. Doyle for several fruitful discussions on this work. This research was supported by ONR MURI N00014-08-1-0747 “Scalable, Data-driven, and Provably-correct Analysis of Networks," ARO MURI W911NF-08-1-0233 "Tools for the Analysis and Design of Complex Multi-Scale Networks," and the Army's W911NF09-D-0001 Institute for Collaborative Biotechnology.

\section{REFERENCES}

[1] M. Huneault and F. D. Galiana, "A survey of the optimal power flow literature," IEEE Transactions on Power Systems, vol. 6, no. 2, pp. 762-770, 1991.

[2] J. Carpentier, "Contribution to the economic dispatch problem," Bulletin Society Francaise Electriciens, vol. 3, no. 8, pp. 431-447, 1962.

[3] G. L. Torres and V. H. Quintana, "Optimal power flow by a nonlinear complementarity method," IEEE Transactions on Power Systems, vol. 15, no. 3, pp. 1028-1033, 2000.

[4] H. Wang, C. E. Murillo-Sanchez, R. D. Zimmerman, and R. J. Thomas, "On Computational issues of market-based optimal power flow," IEEE Transactions on Power Systems, vol. 22, no. 3, pp. 1185-1193, 2007.

[5] Y. Zhu and K. Tomsovic, "Optimal distribution power flow for systems with distributed energy resources," Electrical Power and Energy Systems, vol. 29, no. 3, pp. 260-267, 2007.

[6] D. Das, "Optimal placement of capacitors in radial distribution system using a Fuzzy-GA method," International Journal of Electrical Power and Energy Systems, vol. 30, no. 6-7, pp. 361-367, 2008.
[7] J. S. Savier and D. Das, "Impact of network reconfiguration on loss allocation of radial distribution systems," IEEE Transactions on Power Delivery, vol. 22, no. 4, pp. 2473-2480, 2007.

[8] E. M. Carreno, R. Romero, and A. Padilha-Feltrin, "An efficient codification to solve distribution network reconfiguration for loss reduction problem," IEEE Transactions on Power Systems, vol. 23, no. 4, pp. 1542-1551, 2008.

[9] G. K. Viswanadha Raju and P. R. Bijwe, "An efficient algorithm for minimum loss reconfiguration of distribution system based on sensitivity and heuristics," IEEE Transactions on Power Systems, vol. 23, no. 3, pp. 1280-1287, 2008.

[10] C. L. T. Borges, and D. M. Falcao, "Impact of distributed generation allocation and sizing on reliability, losses and voltage profile," IEEE Bologna Power Tech Conference, Bologna, Italy, 2003.

[11] C. Wang and M. H. Nehrir, "Analytical approaches for optimal placement of distributed generation sources in power systems," IEEE Transactions on Power Systems, vol. 19, no. 4, pp. 2068-2076, 2004.

[12] E. Haesen, M. Espinoza, B. Pluymers, I. Goethals, V. V. Thong, J. Driesen, R. Belmans, and B. D. Moor, "Optimal placement and sizing of distributed generator units using genetic optimization algorithm," Electrical Power Quality and Utilisation, vol. 11, no. 1, pp. 97-104, 2005.

[13] J. B. V. Subrahmanyam and C. Radhakrishna, "Distributed generator placement and sizing in unbalanced radial distribution system," International Journal of Electrical Power and Energy Systems Engineering, vol. 2, no. 4, pp. 232-239, 2009.

[14] S. Boyd and L. Vandenberghe, "Convex Optimization," Cambridge University Press, 2004.

[15] J. Lavaei and S. H. Low, "Zero duality gap in optimal power flow problem," Technical Report, California Institute of Technology, 2010 (available online at http://caltechcdstr.library.caltech.edu/177).

[16] B. Recht, M. Fazel and P. A. Parrilo, "Guaranteed minimum rank solutions to linear matrix equations via nuclear norm minimization," to appear in SIAM Review, 2010.

[17] B. Recht, W. Xu and B. Hassibi, "Null space conditions and thresholds for rank minimization," to appear in Mathematical Programming, 2010.

[18] F. Milano, L. Vanfretti, and J. C. Morataya, "An open source power system virtual laboratory: the PSAT case and experience," IEEE Transactions on Education, vol. 51, no. 1, pp. 17-23, 2008. 